Article

\title{
Serum Anti-Heart and Anti-Intercalated Disk Autoantibodies: Novel Autoimmune Markers in Cardiac Sarcoidosis
}

\author{
Alida L. P. Caforio ${ }^{1, *(\mathbb{C}}$, Anna Baritussio ${ }^{1}$, Renzo Marcolongo ${ }^{2}\left(\mathbb{0}\right.$, Chun-Yan Cheng ${ }^{1}{ }^{\oplus}$, Elena Pontara ${ }^{1}$, \\ Elisa Bison ${ }^{1}$, Maria Grazia Cattini ${ }^{1}$, Nicoletta Gallo ${ }^{3}$, Mario Plebani ${ }^{3}{ }^{[}$, , Sabino Iliceto ${ }^{1}$, Gianpietro Semenzato ${ }^{2}$, \\ Lisa Maier ${ }^{4}$ and Nabeel Hamzeh ${ }^{5}$
}

1 Division of Cardiology, Department of Cardiological Thoracic and Vascular Sciences and Public Health, University of Padova, 35100 Padova, Italy; anna.baritussio@aopd.veneto.it (A.B.); 073047chengchunyan@163.com (C.-Y.C.); elena.pontara@unipd.it (E.P.); elisa.bison@unipd.it (E.B.); mariagrazia.cattini@unipd.it (M.G.C.); sabino.iliceto@unipd.it (S.I.)

2 Hematology and Clinical Immunology, Department of Medicine, University of Padova, 35100 Padova, Italy; renzo.marcolongo@aopd.veneto.it (R.M.); g.semenzato@unipd.it (G.S.)

3 Department of Laboratory Medicine, University of Padova, 35100 Padova, Italy; nicoletta.gallo@aopd.veneto.it (N.G.); mario.plebani@unipd.it (M.P.)

4 Division of Environmental and Occupational Health Sciences, Department of Medicine, National Jewish Health, Denver, CO 80206, USA; maier1@njhealth.org

5 Division of Pulmonary, Critical Care and Occupational Medicine, University of Iowa, Iowa City, IA 52242, USA; nabeel-hamzeh@uiowa.edu

* Correspondence: alida.caforio@unipd.it; Tel.: +39-049-821-2348; Fax: +39-049-821-1802

\section{check for} updates

Citation: Caforio, A.L.P.; Baritussio, A.; Marcolongo, R.; Cheng, C.-Y.; Pontara, E.; Bison, E.; Cattini, M.G.; Gallo, N.; Plebani, M.; Iliceto, S.; et al. Serum Anti-Heart and Anti-Intercalated Disk Autoantibodies: Novel Autoimmune Markers in Cardiac Sarcoidosis. J. Clin. Med. 2021, 10, 2476. https:// doi.org/10.3390/jcm10112476

Academic Editor: Andrea Frustaci

Received: 9 April 2021

Accepted: 31 May 2021

Published: 2 June 2021

Publisher's Note: MDPI stays neutral with regard to jurisdictional claims in published maps and institutional affiliations.

Copyright: (c) 2021 by the authors Licensee MDPI, Basel, Switzerland. This article is an open access article distributed under the terms and conditions of the Creative Commons Attribution (CC BY) license (https:// creativecommons.org/licenses/by/ $4.0 /)$.
Abstract: Background: Sarcoidosis is an immune-mediated disease. Cardiac involvement, a granulomatous form of myocarditis, is under-recognized and prognostically relevant. Anti-heart autoantibodies (AHAs) and anti-intercalated disk autoantibodies (AIDAs) are autoimmune markers in nonsarcoidosis myocarditis forms. Objective: The aim was to assess serum AHAs and AIDAs as autoimmune markers in cardiac sarcoidosis. Methods: This is a cross-sectional study on AHA and AIDA frequency in: 29 patients (aged $46 \pm 12,20$ male) with biopsy-proven extracardiac sarcoidosis and biopsy-proven or clinically suspected and confirmed by 18-fluorodeoxyglucose positron emission tomography and/or cardiovascular magnetic resonance (CMR) cardiac involvement; 30 patients (aged $44 \pm 11,12$ male) with biopsy-proven extracardiac sarcoidosis without cardiac involvement (no cardiac symptoms, normal 12-lead electrocardiogram, echocardiography and CMR), and control patients with noninflammatory cardiac disease (NICD) $(n=160)$, ischemic heart failure (IHF) $(n=141)$ and normal blood donors (NBDs) $(n=270)$. Sarcoidosis patients were recruited in two recruiting tertiary centers in the USA and Italy. AHAs and AIDAs were detected by indirect immunofluorescence on the human myocardium and skeletal muscle. Results: AHA and AIDA frequencies were higher in sarcoidosis with cardiac involvement $(86 \% ; 62 \%)$ than in sarcoidosis without cardiac involvement $(0 \% ; 0 \%)$, NICD $(8 \% ; 4 \%)$, IHF $(7 \% ; 2 \%)$ and NBD $(9 \% ; 0 \%)(p=0.0001 ; p=0.0001$, respectively). Sensitivity and specificity for cardiac sarcoidosis were $86 \%$ and $92 \%$ for positive AHAs and $62 \%$ and $98 \%$ for positive AIDAs, respectively. AIDAs in cardiac sarcoidosis were associated with a higher number of involved organs $(p=0.04)$. Conclusions: Serum AHAs and AIDAs provide novel noninvasive diagnostic autoimmune markers for cardiac sarcoidosis.

Keywords: sarcoidosis; myocarditis; autoimmunity; cardiac autoantibodies

\section{Introduction}

Sarcoidosis is a systemic granulomatous disease of still unknown etiology that can involve any organ or system, including the heart [1-11]. The disease has a variable presentation, followed by either spontaneous remission or chronic remitting-relapsing progression, which usually requires prolonged systemic immunosuppressive therapy $[12,13]$. 
Cardiac involvement is considered a major clinical presentation, as it involves a sizable proportion of patients [1,4,9]; if not promptly recognized and treated, it is associated with poor prognosis [8]. Indeed, cardiac sarcoidosis can have a silent evolution [13] or is unexpectedly present with malignant arrhythmia, leading to sudden cardiac death $[5,10,11]$. Therefore, the identification of reliable biomarkers of early and/or subclinical cardiac involvement, including serum autoantibodies [14,15], would be essential for both early diagnosis and therapeutic intervention, which can reduce morbidity and mortality.

Autoimmunity has been described in sarcoidosis with an increased prevalence of autoimmune thyroiditis [14], and autoantibodies have been detected in sarcoidosis patients with uveitis [15]. There is growing evidence that B-cells and humoral immunity may play a role in sarcoidosis immunopathogenesis [16]. Anti-heart autoantibodies (AHAs) and anti-intercalated disk autoantibodies (AIDAs) are reliable autoimmune markers in nonsarcoidosis myocarditis forms [17-23]. To assess the potential role of AHAs and AIDAs as noninvasive biomarkers of cardiac sarcoidosis, we evaluated the frequency of AHAs and AIDAs in sera from a cohort of 29 patients with a biopsy-confirmed diagnosis of extracardiac sarcoidosis and biopsy-proven or clinically suspected cardiac involvement $[3,23]$, as well as in an age-matched group of 30 patients with a biopsy-confirmed diagnosis of extracardiac sarcoidosis without cardiac involvement.

\section{Methods}

\subsection{Study Patients with Sarcoidosis}

A cohort of 29 sarcoidosis patients with cardiac involvement and a cohort of 30 sarcoidosis patients without cardiac involvement were retrospectively selected from the National Jewish Health Sarcoidosis Biorepository (USA) and the Cardiology Clinic of Padova University Hospital (Italy). Cardiac sarcoidosis diagnosis required fulfillment of the criteria of the Heart and Rhythm Society consensus proposal [10,11]. A diagnosis of probable cardiac sarcoidosis was considered sufficient for study enrolment, and in 2 patients, endomyocardial biopsy was performed. All patients had histological confirmation of extracardiac sarcoidosis. Coronary angiography was not mandatory to exclude coronary artery disease, but coronary artery disease was ruled out in all patients by noninvasive tests, e.g., standard 12-lead electrocardiogram, an electrocardiographic stress test, cardiac magnetic resonance (CMR) and/or coronary computed tomography, and, as clinically indicated, by selective coronary angiography. Patients with relevant comorbidities were excluded (e.g., malignancy, ischemic heart disease, diabetes, peripheral artery disease, stroke, systemic autoimmune diseases).

\subsection{Serum AHAs and AIDAs Testing by Indirect Immunofluorescence (IFL)}

Sera from the sarcoidosis patients with and without cardiac involvement were available and tested, blindly from clinical diagnosis, for AHAs and AIDAs by IFL at 1/10 dilution on $4 \mu \mathrm{m}$ thick, unfixed, fresh-frozen cryostat sections of blood group $\mathrm{O}$ normal human atrium and skeletal muscle [17-23]. Organ-specific and cross-reactive AHA patterns were classified as described [17-23]. Briefly, organ-specific AHAs gave diffuse and/or striational cytoplasmic staining of atrial myocytes but were negative on skeletal muscle (Figure 1C-F). Cross-reactive 1 or partially organ-specific AHAs gave a fine striational staining on the atrium but was negative or only weakly stained skeletal muscle. Cross-reactive 2 AHAs gave a broad striational pattern on longitudinal sections of the heart and skeletal muscle [17]. Absorption studies with relevant tissues have confirmed the organ specificity and cross-reactivity of the AHA patterns [17]. AIDAs gave a linear staining of the intercalated disks between cardiac myocytes (Figure 1E) [22]. Two sera were used as standard positive and negative controls and titrated in every assay. All sera were read blindly against these standards using a fluorescence microscope (Zeiss Axioplan 2 imaging, Zeiss, New York, NY, USA). An additional positive control serum was titrated to assess reproducibility. Endpoint titers for this serum were reproducible within one double dilution in all assays [17-23]. The frequency of AHAs and AIDAs in sarcoidosis was compared with that observed in 
previously established control groups of noninflammatory cardiac disease (NICD) ( $n=160$, 80 male, aged $37 \pm 17$ years, of whom $n=55$ rheumatic heart disease, $n=67$ hypertrophic cardiomyopathies and $n=38$ congenital defects), ischemic heart disease ( $n=141,131$ male, aged $44 \pm 14$ years) and normal individuals $(n=270,123$ male, aged $35 \pm 11)$ [17-23].
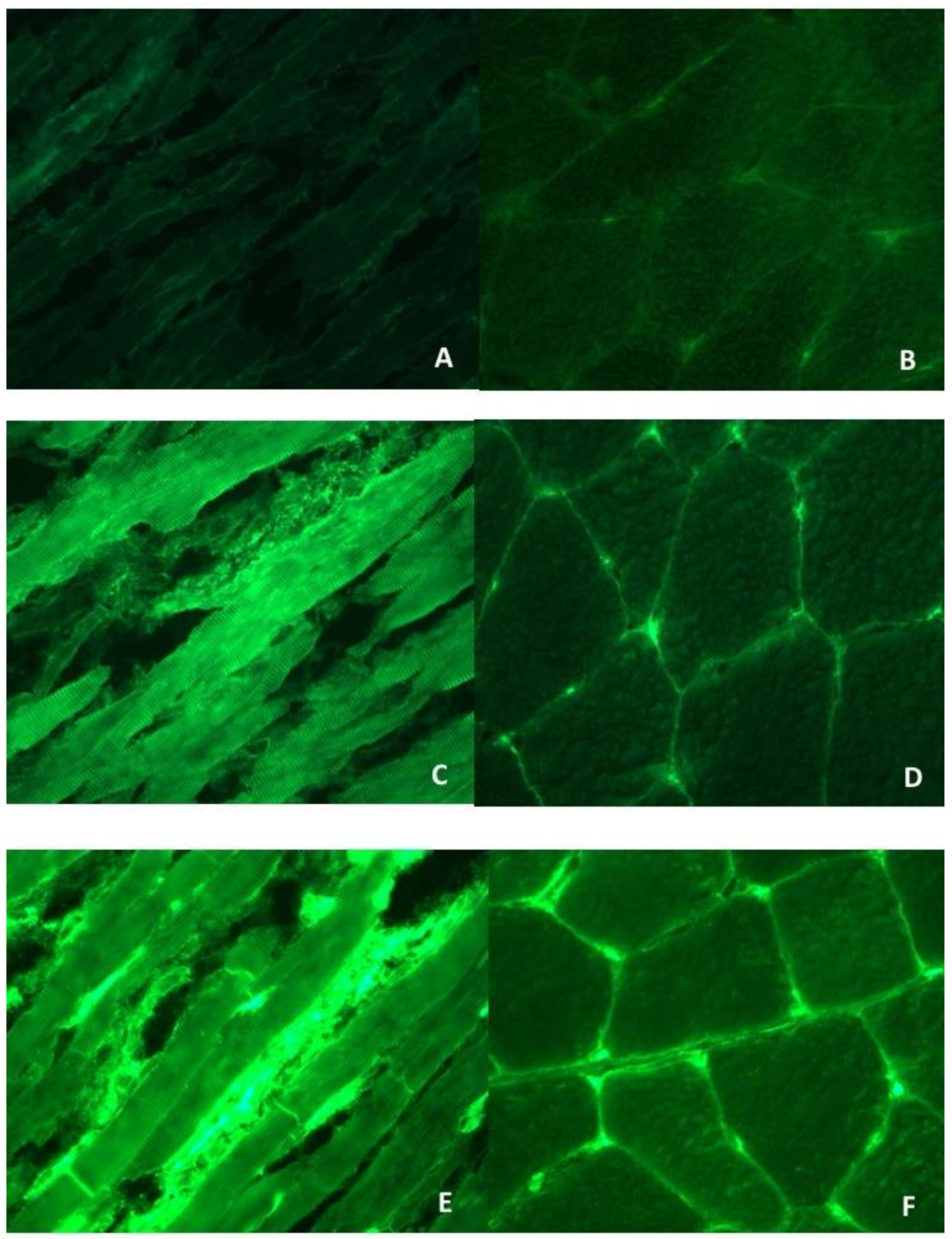

Figure 1. Anti-heart autoantibody (AHA) and anti-intercalated disk autoantibody (AIDA) patterns by indirect immunofluorescence test. Negative AHA and AIDA control serum pattern (A) on human heart tissue (negative $(\times 400)$ ) and $($ B) on human skeletal muscle (negative $(\times 200)$ ). Organ-specific AHA pattern $(\mathbf{C})$ on human heart tissue (strong cytoplasmic and striational staining of cardiac myocytes (organ-specific AHA pattern) $(\times 400)$ ) and $(D)$ on human skeletal muscle tissue (negative $(\times 200)$ ). Organ-specific AHA and AIDA pattern: $(E)$ strong linear staining of the intercalated disks (AIDA pattern) and associated weak organ-specific AHA pattern $(\times 400)$ and $(\mathbf{F})$ on human skeletal muscle tissue (negative $(\times 200)$ ). 


\subsection{Statistical Analysis}

Results for quantitative measures are given as the mean $\pm \mathrm{SD}$ or as the median (interquartile range) for variables deviating from the normal distribution. The Student's $t$-test, one-way analysis of variance, $\chi^{2}$ test, Fisher's exact test, or the Kolmogorov-Smirnov test were used as appropriate. All $p$-values were two-tailed; values of $p<0.05$ indicated statistical significance. All statistical analysis was performed using STATA/IC software version 14.0 (STATA Corp Inc., College Station, TX, USA, 2015).

\section{Results}

\subsection{Clinical, Diagnostic and Imaging Features in Sarcoidosis Patients}

The clinical, diagnostic and imaging features in patients with cardiac sarcoidosis are given in Tables 1-3. At the time of serum sampling, $23(79 \%)$ patients were not on immunosuppressive therapy, 3 (11\%) were on low-dose (range: 7.5 to $20 \mathrm{mg} /$ day) prednisone, $1(3.5 \%)$ on azathioprine and $1(3 \%)$ on hydroxychloroquine; none had a fever or increased serum angiotensin-converting enzyme levels. Age at diagnosis of cardiac involvement was higher than the age at diagnosis of extracardiac disease $(p=0.01)$. The majority of patients were male $(69 \%)$ and Caucasian (76\%). Evidence of lung involvement was present in $86 \%$ of patients. The most common electrocardiographic feature was right bundle branch block (38\%); overall left ventricular ejection fraction (LVEF) was preserved by echocardiography and CMR. However, the majority (87\%) of the patients undergoing CMR had late gadolinium enhancement (LGE) in a nonischemic pattern. In addition, $17 \%$ had evidence of edema on CMR. The most common location of LGE was the interventricular septum $(26 \%)$, and an equal proportion had multiple LGE sites. The most common LGE pattern was spot-like. Finally, multifocal hypermetabolic activity on 18-fluorodeoxyglucose positron emission tomography (FDG-PET) was seen in 33\% of the tested patients $(n=18)$, and the right ventricle was involved in $28 \%$ of tested patients. Overall heart involvement by FDG-PET was found in $67 \%$ of the tested patients. Endomyocardial biopsy was diagnostic in one case from the USA series; in another from Padova, it was not diagnostic.

The 30 sarcoidosis patients without cardiac involvement had a similar age at diagnosis compared to those with cardiac involvement ( $44 \pm 11$ vs. $46 \pm 12$, respectively, $p=\mathrm{NS}$ ), were more commonly female $(18,60 \%$ vs. $9,31 \%$, respectively, $p=0.02)$ and had a similar distribution of extracardiac organ involvement (lung in $28,93 \%$; eye in 3, $10 \%$; liver in 1 , $3 \%$; skin in $4,13 \%$; other organs in $5,17 \%$ ) compared to patients with cardiac involvement (shown in Table 1). Patients without cardiac involvement did not have cardiac symptoms, had normal electrocardiographic, echocardiographic and CMR findings, and all were not taking immunosuppressive drugs at the time of serum sampling, except for one patient on methotrexate.

\subsection{AHA and AIDA Frequency in Sarcoidosis and Association with Clinical and Diagnostic Features}

A representative example of AHA and AIDA patterns is shown in Figure 1. Twentyfive $(86 \%)$ cardiac sarcoidosis sera tested AHA positive, of which $23(92 \%)$ were of the organ-specific pattern; the remaining $2(8 \%)$ were classified as partially organ-specific, and 18 cardiac sarcoidosis sera tested AIDA positive (62\%). The frequencies of AHAs and AIDAs were higher in cardiac sarcoidosis $(86 \% ; 62 \%)$ than sarcoidosis without cardiac involvement $(0,0 \% ; 0,0 \%)$, in NICD $(8 \% ; 4 \%)$, IHF $(7 \% ; 2 \%)$ and NBD $(9 \% ; 0 \%)(p=0.0001$; $p=0.0001$, respectively). Figure 2 shows the receiver operator characteristic (ROC) curve of AHA testing for cardiac sarcoidosis. The best cut-off point was obtained by considering positive both the organ-specific and partially organ-specific AHA patterns, with sensitivity and specificity of $86 \%$ and $95 \%$, respectively. Sensitivity and specificity for positive AIDAs were $62 \%$ and $98 \%$, respectively. 
Table 1. Clinical features of cardiac sarcoidosis patients $(n=29)$.

\begin{tabular}{lc}
\hline Age at diagnosis of sarcoidosis, mean \pm standard deviation & \\
Age at diagnosis of cardiac sarcoidosis, mean \pm standard & $46 \pm 12$ \\
deviation & $49 \pm 11$ \\
Female sex, $n(\%)$ & $9(31)$ \\
Race, $n(\%)$ & \\
Caucasian & $22(76)$ \\
African American & $5(17)$ \\
Hispanic & $2(7)$ \\
Former or active smoker & $8(28)$ \\
Dyspnea, $n(\%)$ & $4(14)$ \\
Chest pain, $n(\%)$ & $3(10)$ \\
Palpitations, $n(\%)$ & $9(31)$ \\
Syncope, $n(\%)$ & $4(14)$ \\
Pericardial effusion, $n(\%)$ & $1(3)$ \\
More than one symptom, $n(\%)$ & $2(7)$ \\
Implantable cardioverter defibrillator, $n(\%)$ & $20(69)$ \\
Arrhythmia at onset, $n(\%):$ & \\
Complete heart block & $1(3)$ \\
Ventricular tachycardia & $1(3)$ \\
Lung involvement, $n(\%)$ & $25(86)$ \\
Eye involvement, $n(\%)$ & $4(14)$ \\
Liver involvement, $n(\%)$ & $3(10)$ \\
Skin involvement, $n(\%)$ & $7(24)$ \\
Small fiber neuropathy, $n(\%)$ & $1(3)$ \\
Pulmonary hypertension, $n$ (\%) & $1(3)$ \\
Other organ involvement, $n(\%)$ & $7(24)$ \\
Number of involved organs, mean \pm standard deviation & $1.8 \pm 1.1$ \\
\hline
\end{tabular}

Table 2. Biochemical, electrocardiographic and echocardiographic features of cardiac sarcoidosis patients $(n=29)$.

\begin{tabular}{lc}
\hline 12-lead electrocardiogram, $n,(\%):$ & \\
Sinus rhythm & $24(83)$ \\
Atrial fibrillation & $2(7)$ \\
Pacemaker & $3(10)$ \\
Atrioventricular block, 1st degree & $3(10)$ \\
Atrioventricular block, 2nd degree & $0(0)$ \\
Atrioventricular block, 3rd degree & $3(10)$ \\
QRS morphology: & \\
Right bundle branch block & $11(38)$ \\
Left bundle branch or left ventricular anterior hemiblock & $3(10)$ \\
Nonspecific intraventricular block & $2(7)$ \\
24 h Holter monitoring $(n=21), n(\%):$ & \\
Frequent ventricular ectopy $(<6 \%$ of total) & $5(24)$ \\
Nonsustained ventricular tachycardia & $4(19)$ \\
Sustained ventricular tachycardia & $2(10)$ \\
2D transthoracic echocardiography: & \\
LVEF, mean \pm standard deviation & $60 \pm 7.6$ \\
Left ventricular hypertrophy, $n(\%)$ & $1(3)$ \\
Left ventricular dilatation, $n(\%)$ & $1(3)$ \\
Left ventricular diastolic dysfunction, $n(\%)$ & $13(46)$ \\
Right ventricular dilatation, $n(\%)$ & $6(21)$ \\
Right ventricular dysfunction, $n(\%)$ & $3(10)$ \\
Left atrial dilatation, $n(\%)$ & $6(21)$ \\
\hline
\end{tabular}


Table 3. Cardiac imaging features of cardiac sarcoidosis patients $(n=29)$.

\begin{tabular}{lc}
\hline Normal coronary angiography, $(n=9){ }^{*}$ remainder not done & $9(100 \%)$ \\
Cardiac magnetic resonance imaging $(n=23):$ & \\
Left ventricular ejection fraction, mean (SD) & $60 \pm 8.2$ \\
Late gadolinium enhancement, present, $n(\%)$ & $20(87)$ \\
Edema, present, $n(\%)$ & $4(17)$ \\
Late gadolinium enhancement, distribution, $n(\%):$ & $6(26)$ \\
Septum & $3(13)$ \\
Anterior wall & $2(9)$ \\
Lateral wall & $1(4)$ \\
Inferior wall & $1(4)$ \\
Right ventricle & $6(26)$ \\
$>1$ location & \\
Late gadolinium enhancement pattern, $n(\%):$ & $9(39)$ \\
Spots & $3(13)$ \\
Intramural striae & $1(4)$ \\
Subepicardial striae & $2(9)$ \\
Subendocardial striae & $19(83 \%)$ \\
Overall heart involvement, $n(\%)$ & \\
Positron emission tomography hypermetabolism $(n=18):$ & $1(6)$ \\
Right atrial, $n(\%)$ & $2(11)$ \\
Left atrial, $n(\%)$ & $1(6)$ \\
Left ventricular septum, $n(\%)$ & $2(11)$ \\
Left ventricular anterior wall, $n(\%)$ & $2(11)$ \\
Left ventricular inferolateral wall, $n(\%)$ & $6(33)$ \\
Left ventricular $>1$ location, $n(\%)$ & $6(33)$ \\
Right ventricle, $n(\%)$ & $12(67)$ \\
Overall heart involvement, $n(\%)$ & \\
\hline
\end{tabular}

* = remainder not done (see Methods section).

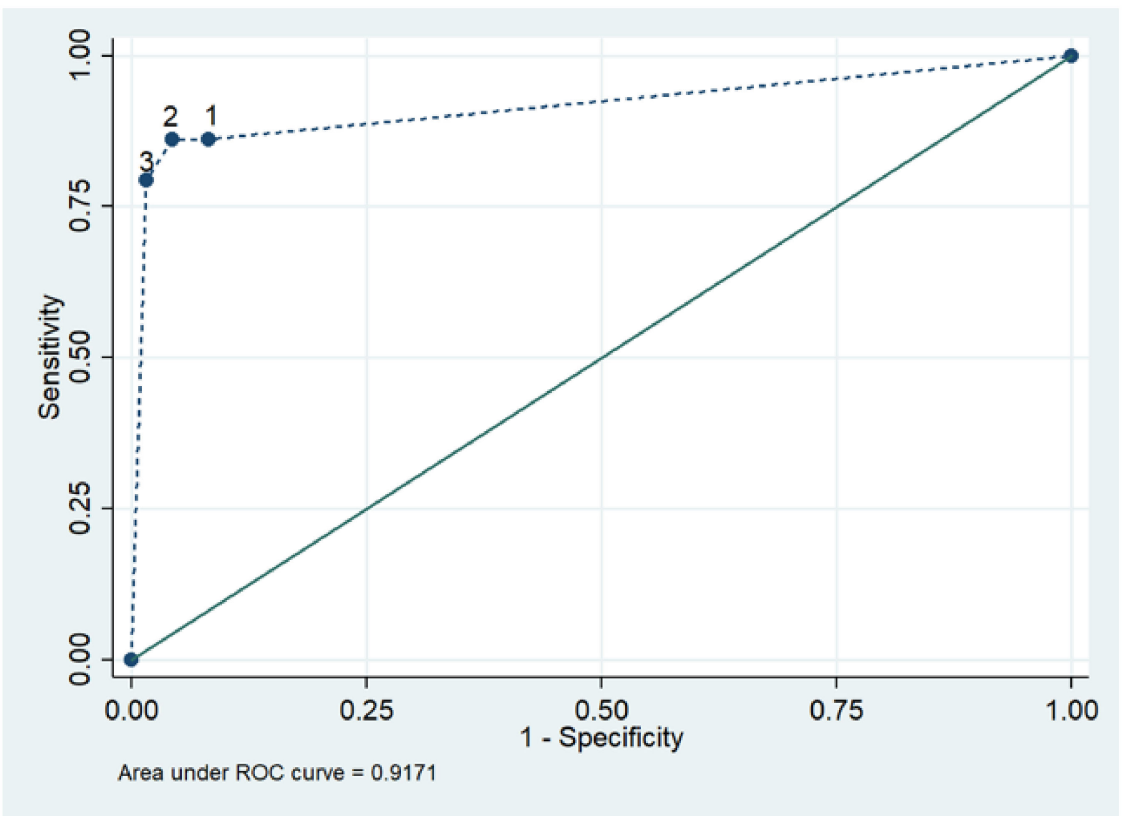

Figure 2. Receiver operating characteristic (ROC) curve of AHA testing in cardiac sarcoidosis. $1=$ considering cross-reactive, partially Specific ( $p$-OS) and organ-specific (OS) AHA patterns as a positive result (sensitivity $(\mathrm{SE})=86.2 \%$; specificity $(\mathrm{SP})=91.8 \%) ; 2=$ considering $p$-OS and OS as a positive result $(\mathrm{SE}=86.2 \%$; $\mathrm{SP}=95.6 \%) ; 3=$ considering only $\mathrm{OS}$ as a positive result $(\mathrm{SE}=79.3 \%$; $\mathrm{SP}=98.4 \%$ ).

After comparison via univariate analysis of the clinical and diagnostic features shown in Tables 1-3 by AHA and AIDA status, positive AIDA status in cardiac sarcoidosis was 
associated with a higher number of involved organs $(2.1 \pm 1.1 \mathrm{vs.} 1.3 \pm 0.9 ; p=0.04)$. A positive AHA status showed a similar trend. Positive AHA and AIDA status was similarly detected in positive and negative PET patients. There were no other statistically significant associations (not shown). Similarly, patients with a high titer $(>1 / 80)$ vs. low $(1 / 10)$ or negative AHAs or AIDAs did not significantly differ in terms of the presence of biochemical, ECG, echocardiographic, MRI or PET abnormalities.

\section{Discussion}

\subsection{Significance and Specificity of AHAs and AIDAs in Cardiac Sarcoidosis}

The main finding of our study was the high frequency of AHAs (86\%) and AIDAs $(62 \%)$ in cardiac sarcoidosis and their absence $(0 \%$ for both AHAs and AIDAs) in sarcoidosis without cardiac involvement. The frequency of AHAs and AIDAs in cardiac sarcoidosis was similar to that found in classical organ-specific nonsarcoidotic autoimmune myocarditis [20,23], supporting the involvement of autoimmunity in cardiac sarcoidosis and in keeping with the growing evidence that B-cells and autoimmunity may play a role in sarcoidosis pathogenesis [24,25]. Importantly, the fact that AHAs and AIDAs were not found in a group of age-matched sarcoidosis patients without cardiac involvement indicates that AHAs and AIDAs are not increased in states of autoimmune activation without myocarditis, such as systemic sarcoidosis. The IFL technique used on the human myocardium and skeletal muscle is standardized, validated and able to distinguish organ-specific cardiac from partially organ-specific (cross-reactive 1 pattern) or fully skeletal muscle cross-reactive AHAs (cross-reactive 2 pattern) [17]. Each assay includes controls for nonspecific antibody binding, and the use of human substrate avoids false-positive reactions due to heterophile antibodies [17]. The organ-specific vs. cross-reactive AHA patterns have previously been confirmed by absorption studies on the heart, skeletal muscle and liver as control [17]; therefore IFL, per se, on a human substrate is able to distinguish cardiac-specific from skeletal muscle cross-reactive AHAs. Recognized autoantigens for AHAs are alpha (entirely cardiac-specific isoform) and beta myosin heavy chain (partially cross-reactive with skeletal muscle) and other unidentified autoantigens by Western blot) [21]. The AIDA pattern is organ-specific; the intercalated disks are specialized cardiac structures, and no AIDAs binding is present on skeletal muscle. Another advantage of IFL is that it detects more than one autoantibody specificity (e.g., AHAs, AIDAs) at the same time, and each autoantibody pattern may relate to more than one autoantigen, as previously shown by Western blotting [21]; thus, it is useful as a screening technique. Conversely, ELISA detects only a single autoantibody specificity, e.g., myosin heavy chain; therefore, it does not replace IFL.

In our cross-sectional cohort, cardiac sarcoidosis was more common among males; similarly, nongranulomatous myocarditis and inflammatory dilated cardiomyopathy are more common in males, possibly reflecting gender-specific susceptibility factors [26]. Our observations lend theoretical support to the wider use of immunosuppressive/immunomodulatory agents in cardiac sarcoidosis, particularly at an early stage, taking into consideration the potential for reducing immune-mediated damage to the myocardium and preventing arrhythmogenic scar formation $[3,23,26]$. This is key in a disease where cardiac involvement is of major prognostic relevance [1-3].

\subsection{AHAs and AIDAs as Potential Diagnostic/Prognostic Markers in Cardiac Sarcoidosis}

Our data show that AHAs and AIDAs may provide useful noninvasive biomarkers, with good sensitivity and specificity for cardiac sarcoidosis (86\% and 95\% for AHAs; $62 \%$ and $98 \%$ for AIDAs, respectively). Interestingly, although three patients were tested while taking low-dose prednisone or azathioprine, all of them turned out to be AHA positive, suggesting that these drugs did not influence the test result. This is promising, as in cardiac sarcoidosis, even the gold standard, endomyocardial biopsy, directed by CMR or electrophysiological mapping, has a particularly reduced sensitivity (50\%), due to the focal nature of sarcoidotic lesions $[3,27,28]$. In keeping with this, in our series, endomyocardial 
biopsy was nondiagnostic in one of the two biopsied patients. Moreover, autopsy studies, surgical heart biopsies taken at the time of left ventricular assist device implantation and, lately, CMR data $[3,7,8]$ have shown clinically silent cardiac involvement in up to $20-25 \%$ of cases, demonstrating that there is a substantial underestimation of cardiac involvement in sarcoidosis and an unmet need for new noninvasive biomarkers [9].

In organ-specific autoimmune myocarditis, the frequency of AHAs and AIDAs is higher in early disease and the antibody titers decline in end-stage chronic inflammatory dilated cardiomyopathy, whereas the antibodies are found in about a third of first-degree apparently healthy relatives and predict disease development at 5 years $[18,19]$. In this study, our population had mostly very mild cardiac sarcoidosis: only three patients had a complete atrioventricular block, two had ventricular tachycardia and the mean LVEF was normal. Thus, the high antibody frequency seen here may relate to the fact that our cohort had early cardiac sarcoidosis. Thus, AHAs and AIDAs may play a role in risk stratifying sarcoidosis patients, especially in silent, subclinical, early cardiac involvement. However, our cross-sectional study design does not allow drawing final conclusions. Future longitudinal studies are warranted to clarify this issue. In nonsarcoidotic myocarditis, AHAs and AIDAs correlate with prognostic features and with disease activity [17-23,29]. In this study, we found no significant associations of antibody status or titer with clinical or diagnostic features including a positive FDG-PET scan, possibly due to the small sample size. Future studies on larger cohorts are needed to clarify the potential of AHAs and AIDAs as prognostic and/or disease activity markers.

\subsection{Cardiac Sarcoidosis, Organ-Specific Myocarditis and Arrhythmogenic Right Ventricular Cardiomyopathy: Evidence for a Common Autoimmune Pathogenetic Pathway}

It is of interest that myosin heavy chain, a relevant autoantigen responsible for the AHA pattern [21], is equally present in the autoantibody recognition of classical organspecific lymphocytic, giant-cell and sarcoidotic myocarditis [23], as well as in arrhythmogenic right ventricular cardiomyopathy [29]. This is not surprising, taking into consideration that myosin is the most abundant protein in the myocardium and that myosin has also been identified as a key autoantigenic target also in postinfectious immune-mediated diseases, such as rheumatic heart disease and Chagas cardiomyopathy [23,30-32]. Similarly, AIDAs and/or other autoantibody specificities to the intercalated disk proteins [33] are found in lymphocytic, giant-cell and sarcoidotic myocarditis, as well as in arrhythmogenic right ventricular cardiomyopathy [33] and in Brugada syndrome [34]. Thus, our findings suggest that in lymphocytic, giant-cell and sarcoidotic myocarditis, as well as in arrhythmogenic right ventricular cardiomyopathy and Brugada syndrome, all highly arrhythmogenic diseases, with similar and often overlapping clinical features, autoimmunity to myosin and intercalated disk proteins may be a common immunopathogenic link. Further work is needed to establish specific autoantigenic targets besides myosin and the potential pathogenic role of AHAs and AIDAs in cardiac sarcoidosis.

In addition, new evidence is emerging for a potential mechanism of T-cell homing to the heart after cardiac insult/injury, which may explain what happens after the autoantibodies bind to cardiac tissue [35]. Such a mechanism may account for cardiotropic immune response directed at a major cardiac autoantigen even in systemic immune-mediated diseases (SIDs) [3], although this requires further exploration.

\subsection{Study Limitations}

A limitation of our study is the lack of biopsy-proven myocardial involvement in all but one patient. However, this is a real-world sample coming from two tertiary centers with expertise in a rare cardiac disease. Endomyocardial biopsy is rarely performed in patients with established extracardiac sarcoidosis, particularly in the United States, and the majority of our patients were followed in the United States. All study patients had noninvasive cardiac imaging confirmation by CMR or FDG-PET. In our patients, AHAs and AIDAs were associated with multiple organ involvement; in addition, the age at diagnosis of cardiac sarcoidosis was higher than the age at diagnosis of systemic disease. 
Nonetheless, this difference may not reflect the natural history of the disease but simply the study design. Work is in progress to prospectively assess the frequency of AHAs and AIDAs in systemic disease without heart involvement, as well as in isolated cardiac sarcoidosis [6], and in relation to newly proposed histopathological surrogate markers [36]. Because cardiac sarcoidosis is more common in Japan than in Western countries $[1,2,4,5]$, it may also be of interest to test AHAs and AIDAs in Japanese patient cohorts.

\section{Conclusions}

In conclusion, the detection of serum AHAs and AIDAs in cardiac sarcoidosis provides novel evidence for the involvement of heart-specific autoimmune reactions in the majority of our cases and a differential diagnostic tool for sarcoidosis without cardiac involvement. Longitudinal studies are needed to clarify whether they may also provide prognostic biomarkers.

Author Contributions: Conceptualization, A.L.P.C., R.M., M.P., S.I., G.S., L.M. and N.H.; Data curation, A.L.P.C., A.B., C.-Y.C., M.G.C., N.G. and N.H.; Formal analysis, A.L.P.C., R.M., E.P. and L.M.; Funding acquisition, E.B.; Investigation, N.G.; Methodology, A.L.P.C., C.-Y.C., E.P., E.B., M.G.C., and N.G.; Supervision, N.H.; Validation, A.L.P.C.; Writing—original draft, A.L.P.C., C.-Y.C., and S.I.; Writing-review \& editing, A.B., R.M., M.P., G.S. and N.H. All authors have read and agreed to the published version of the manuscript.

Funding: A.L.P.C. acknowledges the support of Budget Integrato per la Ricerca dei Dipartimenti (BIRD, 2019), Padova University, Padova, Italy (Project Title: Myocarditis: Genetic Background, Predictors of Dismal Prognosis and of Response to Immunosuppressive Therapy).

Institutional Review Board Statement: The study was conducted according to the guidelines of the Declaration of Helsinki, and approved by the Institutional Review Board of the National Jewish Health, Denver, Colorado USA (Protocol Code: HS2814, date of approval: 21 November 2013, renewed on 28 September 2020) and by the Padova University-Azienda Ospedaliera Ethics Committee, Padova, Italy (Protocol Code: 0027841, date of approval: 6 May 2020).

Informed Consent Statement: Informed consent was obtained from all subjects involved in the study.

Data Availability Statement: Data sharing is not applicable to this article. No new data were created or analyzed in this study.

Conflicts of Interest: The authors declare no conflict of interest.

$\begin{array}{ll}\text { Abbreviations } \\ \text { AHA } & \begin{array}{l}\text { Anti-heart autoantibodies } \\ \text { anti-intercalated disk autoantibodies } \\ \text { AIDA }\end{array} \\ \text { CMR } & \text { cardiac magnetic resonance } \\ \text { FDG-PET } & \text { 18-fluorodeoxyglucose positron emission tomography } \\ \text { IFL } & \text { indirect immunofluorescence } \\ \text { IHF } & \text { ischemic heart failure } \\ \text { LGE } & \text { late gadolinium enhancement } \\ \text { LVEF } & \text { left ventricular ejection fraction } \\ \text { NDB } & \text { normal blood donors } \\ \text { NICD } & \text { noninflammatory cardiac disease }\end{array}$

\section{References}

1. Valeyre, D.; Prasse, A.; Nunes, H.; Uzunhan, Y.; Brillet, P.Y.; Muller-Quernheim, J. Sarcoidosis. Lancet 2014, 383, 1155-1167. [CrossRef]

2. Hamzeh, N.; Steckman, D.A.; Sauer, W.H.; Judson, M.A. Pathophysiology and clinical management of cardiac sarcoidosis. Nat. Rev. Cardiol. 2015, 12, 278-288. [CrossRef] [PubMed]

3. Caforio, A.L.; Adler, Y.; Agostini, C.; Allanore, Y.; Anastasakis, A.; Arad, M.; Böhm, M.; Charron, P.; Elliott, P.M.; Eriksson, U.; et al. Diagnosis and management of myocardial involvement in systemic immune-mediated diseases: A position statement of the European Society of Cardiology Working Group on Myocardial and Pericardial Disease. Eur. Heart J. 2017, 38, $2649-2662$. [CrossRef] [PubMed] 
4. Semenzato, G.; Bortoli, M.; Agostini, C. Applied clinical immunology in sarcoidosis. Curr. Opin. Pulm. Med. 2002, 8, 441-444. [CrossRef]

5. Iwai, K.; Tachibana, T.; Takemura, T.; Matsui, Y.; Kitalchi, M.; Kawabata, Y. Pathological studies on sarcoidosis autopsy. I. Epidemiological features of 320 cases in Japan. Pathol. Int. 1993, 43, 372-376. [CrossRef]

6. Kandolin, R.; Lehtonen, J.; Airaksinen, J.; Vihinen, T.; Miettinen, H.; Ylitalo, K.; Kaikkonen, K.; Tuohinen, S.; Haataja, P.; Kerola, T.; et al. Cardiac sarcoidosis: Epidemiology, characteristics, and outcome over 25 years in a nationwide study. Circulation 2015, 131, 624-632. [CrossRef]

7. Chen, E.S.; Moller, D.R. Etiology of Sarcoidosis. Clin. Chest Med. 2008, 29, 365-377. [CrossRef]

8. Smedema, J.-P.; Snoep, G.; van Kroonenburgh, M.P.; van Geuns, R.-J.; Dassen, W.R.; Gorgels, A.P.; Crijns, H.J. Evaluation of the Accuracy of Gadolinium-Enhanced Cardiovascular Magnetic Resonance in the Diagnosis of Cardiac Sarcoidosis. J. Am. Coll. Cardiol. 2005, 45, 1683-1690. [CrossRef]

9. Broos, C.E.; Van Nimwegen, M.; Hoogsteden, H.C.; Hendriks, R.W.; Kool, M.; Blink, B.V.D. Granuloma Formation in Pulmonary Sarcoidosis. Front. Immunol. 2013, 4. [CrossRef]

10. Birnie, D.H.; Kandolin, R.; Nery, P.B.; Kupari, M. Cardiac manifestations of sarcoidosis: Diagnosis and management. Eur. Heart J. 2016, 38, 2663-2670. [CrossRef]

11. Birnie, D.H.; Sauer, W.H.; Bogun, F.; Cooper, J.M.; Culver, D.A.; Duvernoy, C.S.; Judson, M.A.; Kron, J.; Mehta, D.; Nielsen, J.C.; et al. HRS Expert Consensus Statement on the Diagnosis and Management of Arrhythmias Associated With Cardiac Sarcoidosis. Heart Rhythm. 2014, 11, 1304-1323. [CrossRef]

12. Sadek, M.M.; Yung, D.; Birnie, D.H.; Beanlands, R.S.; Nery, P.B. Corticosteroid Therapy for Cardiac Sarcoidosis: A Systematic Review. Can. J. Cardiol. 2013, 29, 1034-1041. [CrossRef]

13. Nagai, S.; Yokomatsu, T.; Tanizawa, K.; Ikezoe, K.; Handa, T.; Ito, Y.; Ogino, S.; Izumi, T. Treatment with methotrexate and low-dose corticosteroids in sarcoidosis patients with cardiac lesions. Intern. Med. 2014, 53, 2761. [CrossRef]

14. Nakamura, H.; Genma, R.; Mikami, T.; Kitahara, A.; Natsume, H.; Andoh, S.; Nagasawa, S.; Nishiyama, K.; Chida, K.; Sato, A.; et al. High Incidence of Positive Autoantibodies against Thyroid Peroxidase and Thyroglobulin in Patients with Sarcoidosis. Clin. Endocrinol. 1997, 46, 467-472. [CrossRef] [PubMed]

15. Amital, H.; Klemperer, I.; Blank, M.; Yassur, Y.; Palestine, A.; Nussenblatt, R.; Shoenfeld, Y. Analysis of Autoantibodies among Patients with Primary and Secondary Uveitis: High Incidence in Patients with Sarcoidosis. Int. Arch. Allergy Immunol. 1992, 99, 34-36. [CrossRef]

16. Malli, F.; Bargiota, A.; Theodoridou, K.; Florou, Z.; Bardaka, F.; Koukoulis, G.; Gourgoulianis, K.; Daniil, Z. Increased primary autoimmune thyroid diseases and thyroid antibodies in sarcoidosis: Evidence for an under-recognised extrathoracic involvement in sarcoidosis? Hormones 2012, 11, 436-443. [CrossRef]

17. Caforio, A.L.; Bonifacio, E.; Stewart, J.T.; Neglia, D.; Parodi, O.; Bottazzo, G.F.; McKenna, W.J. Novel organ-specific circulating cardiac autoantibodies in dilated cardiomyopathy. J. Am. Coll. Cardiol. 1990, 15, 1527-1534. [CrossRef]

18. Caforio, A.; Keeling, P.; McKenna, W.; Mann, J.; Bottazzo, G.; Zachara, E.; Mestroni, L.; Camerini, F. Evidence from family studies for autoimmunity in dilated cardiomyopathy. Lancet 1994, 344, 777. [CrossRef]

19. Caforio, A.L.; Mahon, N.G.; Baig, M.K.; Tona, F.; Murphy, R.T.; Elliott, P.M.; McKenna, W.J. Prospective familial assessment in dilated cardiomyopathy: Cardiac autoantibodies predict disease development in asymptomatic relatives. Circulation 2007, 115, 76-83. [CrossRef]

20. Caforio, A.L.; Calabrese, F.; Angelini, A.; Tona, F.; Vinci, A.; Bottaro, S.; Ramondo, A.; Carturan, E.; Iliceto, S.; Thiene, G.; et al. A prospective study of biopsy-proven myocarditis: Prognostic relevance of clinical and aetiopathogenetic features at diagnosis. Eur. Heart J. 2007, 28, 1326-1333. [CrossRef]

21. Caforio, A.L.; Grazzini, M.; Mann, J.M.; Keeling, P.J.; Bottazzo, G.F.; McKenna, W.J.; Schiaffino, S. Identification of alpha- and beta-cardiac myosin heavy chain isoforms as major autoantigens in dilated cardiomyopathy. Circulation 1992, 85, 1734-1742. [CrossRef] [PubMed]

22. Caforio, A.L.P.; Brucato, A.; Doria, A.; Brambilla, G.; Angelini, A.; Ghirardello, A.; Bottaro, S.; Tona, F.; Betterle, C.; Daliento, L.; et al. Anti-heart and anti-intercalated disk autoantibodies: Evidence for autoimmunity in idiopathic recurrent acute pericarditis. Heart 2010, 96, 779-784. [CrossRef] [PubMed]

23. Caforio, A.L.P.; Pankuweit, S.; Arbustini, E.; Basso, C.; Gimeno-Blanes, J.; Felix, S.B.; Fu, M.; Heliö, T.; Heymans, S.; Jahns, R.; et al. Current state of knowledge on aetiology, diagnosis, management, and therapy of myocarditis: A position statement of the European Society of Cardiology Working Group on Myocardial and Pericardial Diseases. Eur. Heart J. 2013, 34, $2636-2648$. [CrossRef] [PubMed]

24. Ramstein, J.; Broos, C.E.; Simpson, L.J.; Ansel, K.M.; Sun, S.A.; Ho, M.E.; Woodruff, P.G.; Bhakta, N.R.; Christian, L.; Nguyen, C.P.; et al. IFN- $\gamma$-Producing T-Helper 17.1 Cells Are Increased in Sarcoidosis and Are More Prevalent than T-Helper Type 1 Cells. Am. J. Respir. Crit. Care Med. 2016, 193, 1281-1291. [CrossRef] [PubMed]

25. Kamphuis, L.S.; Van Zelm, M.C.; Lam, K.H.; Rimmelzwaan, G.F.; Baarsma, G.S.; Dik, W.A.; Thio, H.B.; Van Daele, P.L.; Van Velthoven, M.E.; Batstra, M.R.; et al. Perigranuloma localization and abnormal maturation of B cells: Emerging key players in sarcoidosis? Am. J. Respir. Crit. Care Med. 2013, 187, 406-416. [CrossRef] 
26. Tschöpe, C.; Ammirati, E.; Bozkurt, B.; Caforio, A.L.P.; Cooper, L.T.; Felix, S.B.; Hare, J.M.; Heidecker, B.; Heymans, S.; Hübner, N.; et al. Myocarditis and inflammatory cardiomyopathy: Current evidence and future directions. Nat. Rev. Cardiol. 2021, 18, 169-193. [CrossRef]

27. Liang, J.J.; Hebl, V.B.; DeSimone, C.V.; Madhavan, M.; Nanda, S.; Kapa, S.; Maleszewski, J.J.; Edwards, W.D.; Reeder, G.; Cooper, L.T.; et al. Electrogram guidance: A method to increase the precision and diagnostic yield of endomyocardial biopsy for suspected cardiac sarcoidosis and myocarditis. JACC Heart Fail 2014, 2, 466-473. [CrossRef]

28. Nery, P.B.; Keren, A.; Healey, J.; Leug, E.; Beanlands, R.S.; Birnie, D.H. Isolated Cardiac Sarcoidosis: Establishing the Diagnosis With Electroanatomic Mapping-Guided Endomyocardial Biopsy. Can. J. Cardiol. 2013, 29, 1015.e1. [CrossRef]

29. Caforio, A.L.; Re, F.; Avella, A.; Marcolongo, R.; Baratta, P.; Seguso, M.; Gallo, N.; Plebani, M.; Izquierdo-Bajo, A.; Cheng, C.Y.; et al. Evidence from family studies for autoimmunity in arrhythmogenic right ventricular cardiomyopathy: Associations of circulating anti-heart and anti-intercalated disk autoantibodies with disease severity and family history. Circulation 2020, 141, 1238-1248. [CrossRef]

30. Cooper, L.T., Jr.; Hare, J.M.; Tazelaar, H.D.; Edwards, W.D.; Starling, R.C.; Deng, M.C.; Menon, S.; Mullen, G.M.; Jaski, B.; Bailey, K.R.; et al. Giant Cell Myocarditis Treatment Trial Investigators. Usefulness of immunosuppression for giant cell myocarditis. Am. J. Cardiol. 2008, 102, 1535-1539. [CrossRef]

31. Cunningham, M.W. Turning point in myocarditis. Circ. Res. 2009, 105, 403-405. [CrossRef]

32. Marin-Neto, J.A.; Cunha-Neto, E.; Maciel, B.C.; Simões, M.V. Pathogenesis of chronic Chagas heart disease. Circulation 2007, 115, 1109-1123. [CrossRef]

33. Chatterjee, D.; Fatah, M.; Akdis, D.; Spears, D.A.; Koopmann, T.T.; Mittal, K.; Rafiq, M.A.; Cattanach, B.M.; Zhao, Q.; Healey, J.S.; et al. An autoantibody identifies arrhythmogenic right ventricular cardiomyopathy and participates in its pathogenesis. Eur. Heart J. 2018, 39, 3932-3944. [CrossRef]

34. Chatterjee, D.; Pieroni, M.; Fatah, M.; Charpentier, F.; Cunningham, K.S.; Spears, D.A.; Chatterjee, D.; Suna, G.; Bos, J.M.; Ackerman, M.J.; et al. An autoantibody profile detects Brugada syndrome and identifies abnormally expressed myocardial proteins. Eur. Heart J. 2020, 41, 2878-2890. [CrossRef]

35. Komarowska, I.; Coe, D.; Wang, G.; Haas, R.; Mauro, C.; Kishore, M.; Cooper, D.; Nadkarni, S.; Fu, H.; Steinbruchel, D.A.; et al Hepatocyte Growth Factor Receptor c-Met Instructs T Cell Cardiotropism and Promotes T Cell Migration to the Heart via Autocrine Chemokine Release. Immunity 2015, 42, 1087-1099. [CrossRef]

36. Honda, Y.; Nagai, T.; Ikeda, Y.; Sakakibara, M.; Asakawa, N.; Nagano, N.; Nakai, M.; Nishimura, K.; Sugano, Y.; Ohta-Ogo, K.; et al. Myocardial Immunocompetent Cells and Macrophage Phenotypes as Histopathological Surrogates for Diagnosis of Cardiac Sarcoidosis in Japanese. J. Am. Heart Assoc. 2016, 5, e004019. [CrossRef] 